\title{
The role of movement in perceptual adaptation to a tilted retinal image
}

\author{
ARIEN MACK ${ }^{2}$ \\ YESHIVA UNIVERSITY
}

Perceptual adaptation to prismatically created tilt of the retinal image was measured after three conditions of exposure: active, passive and no movement of the observer. No difference in magnitude of effect was found between the two movement conditions. The effects of no movement were considerably less than those attained with movement, thus indicating the effectiveness of movement-produced information for adaptation. An explanation is suggested for the no movement effect in terms of a Wertheimer righting-of-the-field.

The role of movement of the observer in adaptation to a distorted visual field has been a matter of considerable controversy. The experiment to be described is concerned with this problem in adaptation to a tilted visual field.

In an experiment by Mikaelian and Held (1964) it was reported that actively moving subjects exposed to a visual field tilted prismatically 20 degrees adapted, whereas the same subjects when moved passively either adapted much less or not at all. This apparent difference between actively and passively moving subjects is predicted from Held's sensorimotor theory of adaptation in which it is maintained that reafferent stimulation, a correlate of voluntary or active movement, is necessary for adaptation.

However, if the adaptation process is understood to be one involving a perceptual rather than a sensorimotor change, this difference between active and passive observers is no longer either predictable or acceptable. Given this view of adaptation, the necessary condition for its occurrence is the availability of information to the effect that the apparently tilted visual field is not tilted and that therefore directions on the retina have new spatial significance. There is no obvious reason why the relevant sensory information is not equally available to both actively moving and passively moved observers.

This may be seen more clearly by example. If a subject looks through prisms which tilt the visual field counter clockwise (ccw), then all lines within his field will be tilted in that direction. If the scene at which he is looking is a familiar one, a room or a hallway, he will almost always immediately perceive the distortion. If the scene is unfamiliar or somehow ambiguous, and the subject does not move, he has no way of learning that what he sees is not what is actually before him. But this situation begins to change as soon as he moves or is moved, for now there will be sensory information that what appears tilted actually is not. If the subject were to sit down from a standing position or be moved downward, an apparently tilted vertical line would maintain its position on his retina instead of moving at an angle out of his field as in the case of an objectively tilted line. This is so because the actual line is in fact vertical. Similarly if the subject moves or is moved forward in a corridor, a horizontal line running down one wall of that corridor which appears tilted by virtue of the prismatic distortion will also maintain its position on his retina. Again this is so because the line is objectively horizontal and not tilted. And further, any movement in a familiar environment will elicit information that will confirm the initial perception that the apparent tilt is not an objective one.

It is information of this kind which is essential to the process of adaptation, because it is information of this kind which is necessary for the formation of new correlations between retinal orientation and perceived direction. There is, however, one important proviso which must be added. This information will not be present in situations like the ones described unless the subject is aware of the direction in which he is moving or being moved. For, if in the first instance, the subject believes that he is moving downward in a direction congruent with the apparent tilt, or, if in the second instance, he believes that he is moving upwards along a peculiarly inclined floor, the visual information necessary for visual adaptation will now not be available. The fact that in both instances tilted lines maintain their retinal positions will now be accounted for by the direction of the subject's own movement. Therefore in order for the appropriate movement-dependent information to be present, there must be no ambiguity or confusion about the direction of movement. In the situation in which subjects are moved or move through a normal environment there is no reason to expect that the gravitational and proprioceptive information about the direction of movement will not be adequate.

Based on this reasoning, it is difficult to understand why Mikaelian and Held failed in one experiment to obtain evidence of adaptation under conditions of passive exposure and why in another experiment they found active movement yielded a significantly greater effect. It was therefore deemed important to repeat their experiment.

On the basis of the view of adaptation suggested here, it is predicted that moving subjects exposed to a prismatically tilted field will adapt regardless of whether the movement is active or passively im- 
posed, and furthermore that there will be no significant difference between the magnitude of visual change attained under these conditions. Both conditions of exposure should lead to a visual, adaptive change such that, if adaptation is complete, only a line tilted in the direction of the prism tilt and by that amount should appear to be vertical when viewed with prisms removed. The experiment performed provided a test of this hypothesis. In overall design it resembles the experiment by Mikaelian and Held except that it included a third condition which made it possible to distinguish the effectiveness of movement information from that of the sight of a familiar scene. As noted above, sight of a scene such as a corridor is itself a possible source of information and, since it is present for moving observers, it is necessary to ascertain the contribution this information makes to whatever adaptation is obtained.

\section{Method}

The three exposure conditions were therefore: active movement, passive movement, and no movement (stationary). The measure of adaptation was the same in all conditions and consisted of having the subject, in the dark, set a 5 in. electro-luminescent rod, which was at a distance of 121 in., so that it appeared to be vertical. The subject was able to do this by simply pulling on one of two strings placed in his hands which moved the rod either clockwise (cw) or ccw. During all testing the subject used a teeth mold which insured the fact that his head remained still and always in the same position, namely, upright. A circular protractor placed behind the rod at its center of rotation indicated to the experimenter the tilt of the rod. All judgments of the vertical were made without prisms so that what was measured was an aftereffect of prism exposure. Four judgments of the vertical were made prior to and immediately following prism exposure, and at these times the initial positions of the rod, whether cw or ccw, were counterbalanced. Only the eye used during exposure, the right eye, was used during the test. The left eye was occluded throughout the experiment. The difference between the preand post exposure settings was the index of adaptation. The optical device worn during exposure consisted of a pair of right angle prisms mounted one in front of the other and set in a rigid goggle frame. It provided a monocular field of view 30 by 20 degrees, and was set to create either a 40 degree cw or ccw rotation of the visual field. It should be noted that the prismatic visual field here is substantially larger than that provided in the Mikaelian and Held study and the imposed tilt is twice as great. Both of these changes are reason to expect a greater adaptation. ${ }^{3}$ In all three exposure conditions the prisms were worn for a total of $30 \mathrm{~min}$. There was $15 \mathrm{~min}$. of exposure followed by a brief rest in which the subject sat in the dark with eyes closed and then another $15 \mathrm{~min}$. of exposure. During passive exposure the subject stood on a small cart specially designed for this experiment and was pushed up and down the corridors of the laboratory by the experimenter who also steered the cart by means of a steering rod connected to the front wheel. A bite bar was positioned at the front end of the cart and was used to prevent head movement during both active and passive exposure. This insured the fact that passive subjects were fully passive and that the two conditions were comparable, differing only with respect to the voluntary quality of their forward movement. ${ }^{4}$ During active exposure the floor of the cart was removed and the subject walked within the cart, pushing it before him while simultaneously steering it. In the stationary condition the subject sat on a chair raised off the floor and placed at one end of the corridor so that the view from this position was approximately what it had been for moving subjects. The subject's chin was placed on a chin rest and he was instructed not to move. The chin rest was considered as effective a means of stabilizing the head in this condition as the bite bar in the movement conditions, since in this condition the subject did not have to keep his head still while being moved. If, however, the chin rest introduced slight head movements, this would have tended to increase rather than diminish the after-effect.

\section{Subjects}

A total of 16 college and graduate students were tested. All of them participated in both the active and passive conditions while only 10 of them, chosen at random, took part in the stationary condition. Eight of these subjects were first exposed actively and of these eight, four were exposed to a $\mathrm{cw}$ and four to a ccw rotation of the field. For the remaining eight subjects the passive condition was first and again four of these subjects were exposed to a $\mathrm{cw}$ and four to a ccw tilt of the field. For all subjects the stationary condition was the final one, and for all subjects the direction of prism tilt remained the same throughout the experiment. The exposure conditions were separated by at least two days in order to minimize the influence of one condition on the following one.

\section{Results}

The results support the proposed view of adaptation. Both passive and active exposure produced significant adaptation, and the slight difference between the two means which favors passive exposure is not significant. The mean aftereffect in the active condition was 3.6 degrees in the predicted direction (significantly different from 0 at better than the .001 level). For the passive condition the mean was 3.7 which is also significant at better than the .001 level. There were three cases in the active condition and only two in the passive condition in which the mea- 
sured aftereffect was non-adaptive. An interesting side light of these results is that the aftereffect was almost always greatest in the initial condition. There were only two exceptions and these occurred when active preceded passive exposure. The reason for this is by no means clear and is difficult to explain in terms of any theory of adaptation. It does certainly indicate, however, that there was no positve carry over from one condition to the next. Another interesting side light is the rather high correlation between the active and passive conditions of exposure such that a subject who showed a substantial adaptation effect in one condition tended to show a similar effect in the other condition $(r=.78)$.

The mean aftereffect in the stationary condition was 1.1 degree. ${ }^{5}$ This effect is considerably less than the effects obtained under conditions of movement exposure indicating the effectiveness of movement-produced information for adaptation. (The difference between stationary and passive exposure is significant at the .05 level when calculated by the Wilcoxon Matched Pairs Signed Rank Test. Using the same test, the difference between the effects of stationary and active exposure is almost significant at the .05 level.) The fact that this condition produced a significant aftereffect, however, requires an explanation in terms of the theory of adaptation proposed. But it must also be noted that this effect cannot be subsumed under a sensorimotor theory of adaptation since it was attained under conditions involving no movement at all and a control experiment has indicated that it cannot be attributed to a Gibson normalization effect. 6

\section{Discussion}

The effect of a tilted frame or a tilted room on the appearance of a vertical line was described by Wertheimer (1912) and widely investigated by Asch and Witkin (1948). The appearance of the corridor when seen, as in this experiment, through prisms which tilt the visual field is identical to the appearance of an actually tilted corridor as long as the observer is stationary. Thus the conditions of stationary exposure are appropriate for eliciting a Wertheimer effect and what may be called a Wertheimer aftereffect. If the Wertheimer effect were complete, then during exposure, only a line that is aligned with or close to the main upright lines of the field will appear vertical, and if there is an aftereffect, only a line tilted away from the gravitational vertical in the direction in which the field had been tilted will appear vertical. ${ }^{7}$ The predicted direction of this aftereffect is identical to that predicted in prism adaptation to a tilted visual field. It is therefore possible that the aftereffect achieved in the stationary condition is a Wertheimer aftereffect. This effect can be considered to be in the same class of effects as movement-produced adaptation only in so far as the observer in this situation can obtain information that the apparently tilted field is not tilted and therefore that a new retinal direction signifies verticality. This information is available to the extent that the observer "knows" that the visible corridor resembles all others in his experience and is therefore not actually tilted and also to the extent that he "knows" that he is viewing from an upright position. If the observer believed himself to be tilted in a direction opposite to that which the field is actually tilted, the visual situation should, of course, be identical to the one experienced by a person viewing a scene without prisms with his head tilted and there would then be no reason to expect any change in the meaning of retinal direction to occur.

Since this experiment was not designed to distinguish between a Wertheimer aftereffect and prism adaptation, the exact nature of the present effect cannot be further specified. It is, however, important to recognize that part of the effect attained under conditions of movement exposure, where the visible field is tilted with respect to the gravitational vertical, may be attributable to a Wertheimer righting-of-thefield effect, a point that has not been made in previous research on this problem. The fact that Mikaelian and Held report that subjects moving through an environment containing only dimly lit luminous spheres adapt far less than subjects moving through a normal environment may be at least partially accounted for by the absence of a Wertheimer aftereffect in this artificial environment containing no visible lines. The fact that passively moved subjects do not adapt at all under these conditions is once again not readily explained although it may have been the result of a lack of information concerning the direction of movement in this situation, a lack not suffered by actively moving subjects.

There is then no apparent explanation for the difference between the results reported here and those reported by Mikaelian and Held. The changes in apparatus and procedure incorporated in this experiment, e.g., increased prismatic tilt and increased size of the prismatic field of view cannot account for this difference, for any effect of these changes should have been in the direction of increasing rather than diminishing the aftereffects. The fact that subjects in this experiment received only a half hour of prism exposure as compared to the hour-long exposure in the Mikaelian and Held experiment can also not account for the difference in results, for similar experiments on adaptation to prismatic tilt done in the Yeshiva laboratory have indicated no difference in the size of the aftereffect following half hour and hour-long prism exposure. 8 Furthermore if shortening the duration of prism exposure should diminish the aftereffect, it would be expected to do so in all conditions and therefore cannot account for the fact that passive and active exposure here yielded equiva- 
lent aftereffects. In this regard it should be noted that the mean aftereffect of active exposure reported $\left(3.6^{\circ}\right)$ is considerably less than that reported by Mikaelian and Held $\left(6.8^{\circ}\right)$. This difference is again not easily explained. Without evidence to the contrary it might have been attributed to the shortened exposure time. The results of this experiment therefore would seem to be evidence against a reafference theory of adaptation which predicts a significant difference between active and passive prism exposure and evidence for the importance of movement-produced information for visual adaptation, this information being equally available to actively and passively moving subjects.

\section{References}

Asch, S. E., \& Witkin, H. A. Studies in space orientation I \& II .J. exp. Psychol, , 1948, 38, 325-337, 455-477.

Cohen, W., \& Tepas, D. Temporal factors in the perception of verticality. Amer. J. Psychol., 1958, 71, 760-763.

Ebenholtz, S. M. Adaptation to a rotated visual field as a function of degree of tilt and exposure time. J. exp. Psychol., in press.

Ebenholtz, S. M. Transfer of adaptation as a function of interpolated optical tilt to the ipsilateral and contralateral eye. J. exp. Psychol., in press.

Mikaelian, H., \& Held, R. Two types of adaptation to an opticallyrotated visual field. Amer. J. Psychol., 1964, 77, 257-263.

Morant, R. B., \& Beller. H. Adaptation to prismatically rotated visual fields. Science, 1965, 148, 530-531.

Morant, R. B., \& Aronoff, J. Starting position, adaptation \& visual framework as influencing the perception of verticality. $J$. exp. Psychol., 1966, 71, 684-686.

Wertheimer, M. Experimentelle studien über das sehen von bewegung. Z. Psychol., 1912, 61, 161-265.

\section{Notes}

1. This research was done while the author was working as research associate with Dr. Irvin Rock and was made possible by a grant from the National Science Foundation (GB 3410). For a more complete statement of the point of view outlined in this paper see; I. Rock, The nature of perceptual adaptation. New York: Basic Books, 1966. The author wishes to express her gratitude to Dr. Rock for his steady guidance and patient instruction.

2. Now at New School for Social Research Graduate Faculty of Political and Social Science.

3. In a recently reported experiment by $\mathrm{s}$. Ebenholtz it was found that "the magnitude of adaptation was a linear function of optical tilt." (Ebenholtz, in press)

4. In the Mikaelian and Held study neither passive nor active subjects were constrained by a bite bar.

5. This finding is slightly less than that reported by Morant and Beller in an experiment involving similar exposure conditions. (Morant \& Beller, 1965).

6. The experiment referred to is one in which subjects sat looking at a circular grid of lines tilted to the same degree as was the prism field for a period of time comparable to prism exposure. At the end of one half hour there was no consistent effect on the judgment of the vertical thus indicating that no Gibson effect was present during prism exposure. Had it been, it would have been revealed by this control experiment. Further evidence that the effect obtained in the stationary condition is not merely a normalization effect is found in the fact that the effect for many subjects was substantially greater than is usually associated with a Gibson effect.

7. Two experiments which investigated the question of whether or not the exposure to a tilted visual field produced an aftereffect report contradictory findings. (Cohen \& Tepas, 1958) and (Morant \& Arnoff, 1966).

8. Data reported in an experiment by $\mathrm{S}$. Ebenholtz indicates that adaptation to a $10^{\circ}$ tilt of the visual field is no greater following a one hour exposure period than following a half-hour exposure period. (Ebenholtz, in press).

(Accepted for publication December 12, 1966.) 